\title{
Separation of Copper and Nickel by Solvent Extraction Using LIX 664N
}

\author{
Rashmi Kumar ${ }^{1}$, Dahyalal J. Shah ${ }^{2}$, Krishna Kant Tiwari ${ }^{3}$ \\ ${ }^{1}$ D. J. Sanghvi College of Engineering, Mumbai, India; ${ }^{2}$ Mukesh Patel School of Technology Management \& Engineering (NMIMS), \\ Mumbai, India; ${ }^{3}$ Jaypee University of Engineering and Technology (JUET), Madhya Pradesh, India. \\ Email: rashmikumar_djs@yahoo.co.in
}

Received February $11^{\text {th }}$, 2013; revised March $13^{\text {th }}, 2013$; accepted April $12^{\text {th }}, 2013$

Copyright (C) 2013 Rashmi Kumar et al. This is an open access article distributed under the Creative Commons Attribution License, which permits unrestricted use, distribution, and reproduction in any medium, provided the original work is properly cited.

\begin{abstract}
Separation of copper and nickel by liquid-liquid extraction from aqueous solutions was studied using LIX 664N in kerosene as solvent. Both metals were taken in their sulfate form and ratio of copper to nickel in feed solutions was maintained as 10:1. LIX $664 \mathrm{~N}$ concentration in kerosene was varied from $10 \%$ to $40 \%(\mathrm{v} / \mathrm{v})$ and its effect was studied on percent extraction of copper and nickel for organic to aqueous (O:A) phase ratio of 2:1 at pH 2. Experimental results showed that at $\mathrm{pH} 2$, copper was selectively extracted from its mixture with nickel. Selectivity for copper extraction was as high as 6000 with $40 \%$ LIX 664N. Calculations using extraction isotherm indicate almost complete recovery of copper in two stages with O:A ratio of 1:1 and in one stage with O:A ratio of 2:1. After extraction of copper, the pH of raffinate containing nickel was adjusted to a value of 9 by addition of ammonia solution. The effect of LIX 664N concentration on extraction of nickel was studied. Up to $80 \%$ nickel could be extracted with 30\% LIX 664N at O:A phase ratio of 2:1. Stripping of copper from the organic phase with $180 \mathrm{~g} / \mathrm{l}$ sulfuric acid at O:A phase ratio of 1:1 gave 98.5\% copper recovery in a two-stage operation.
\end{abstract}

Keywords: Copper; Nickel; LIX 664N; Solvent Extraction; Distribution Coefficient; Selectivity

\section{Introduction}

Due to an increasing demand for metals, continuous research into finding more effective and efficient methods for recycling of previously used metals is essential. There is an urgent need for economic recovery of the metals in an environment friendly way. The ever increasing quantities of waste electric and electronic equipment are producing huge quantities of waste printed circuit boards (pcbs).

The recovery and separation of metals in discarded pcbs have become a necessity not only to reuse the metals but also to protect the environment from contamination with the toxic metals. Printed circuit boards are a heterogeneous mixture of base metals such as iron, aluminum, copper, zinc and nickel and precious metals such as gold, silver, platinum, etc. [1-3].

For recovery of metals from discarded pcbs, the various components on the printed circuit boards are first dismantled by manual, automated or combination of both methods [4]. The disassembled parts and components are categorized into reusable components, environmentally hazardous and those with noble metals. For recovery of tin, tin alloys or lead alloys such as solder, leaching technology can be used to dissolve the solder and then the dissolved solder can be recovered in pure metallic form by electrolytic processes [5]. For efficient liberation and separation of metallic components from non-metallic components such as plastics and ceramics, the pcbs are initially crushed. Grinding is followed by material separation based on physical properties namely magnetic, electrostatic, density, visual, etc.

Further retrieving of metals can be done either by pyrometallurgical or hydrometallurgical processes. The pyrometallurgical route has been adopted by a few countries and smelters are successfully used for retrieving of various metals [6]. Many alternative hydrometallurgical routes are also developed for the efficient separation of metals in a cost-effective and environment-friendly manner. Some papers have reported a promising method for separation of metals in pcbs by solvent extraction $[7,8]$. For separation of metals by solvent extraction, the pcb fines are first to be leached in a suitable solvent and then the metals can be separated using various extractants.

Copper constitutes approximately 15 - 20 weight $\%$ of 
the total metal content in a pcb and nickel though constitutes a small weight\% has a high economic value. Hence in this study experiments on separation of copper and nickel by solvent extraction have been performed using a modified aldoxime LIX $664 \mathrm{~N}$ as the extractant. LIX reagents are chelating extractants. The development of LIX reagents (Liquid Ion Exchange Reagents) for extraction of metals has been a major breakthrough in efficient and economical separation of metals by solvent extraction. There are several LIX reagents such as LIX 84, LIX 984, LIX 984N, LIX 622, LIX 622N, LIX 973, LIX $973 \mathrm{~N}$ that are available for copper extraction. The "N" products have different chemical structures than the others. As a result, "N" products have slightly faster kinetics (rate of reaction). However, the "N" products have slightly lower stability factors due to their difference in chemical composition [9].

In the papers by Alguacil and Cobo [10] and Sridhar et $a l$. [11] it has been shown that copper and nickel can be separated using LIX 973N and LIX 984N, respectively by co-extraction from ammoniacal/ammonium carbonate aqueous media followed by their selective stripping. But there can be appreciable ammonia carryover by the organic phase from the solvent feed to strip as suggested by Flett and Melling [12]. Another method of separation of copper and nickel has been proposed by Giannopoulou and Panias[13] by electro recovery of copper followed by nickel precipitation through neutralization.

In this work separation of copper and nickel is carried by selective extraction of copper and nickel at different $\mathrm{pH}$ followed by the stripping of the respective loaded organic phases for recovery of copper and nickel. The extractant used is LIX 664N.

LIX 664N is a mixture of 5-nonylsalicylaldoxime with a proprietary ester modifier and a high flash point hydrocarbon diluent. Salicylaldoximes provide high copper transfer kinetics and extractive strength. Since not much published data is available for LIX 664N, it has been chosen as the extractant for separating copper and nickel in this study.

\section{Experimental}

\subsection{Materials and Methods}

The simulated feed solution had both copper and nickel in sulfate medium in a ratio of 10:1, approximately the same as that in a pcb. Commercial grade kerosene was used as diluent. LIX 664N was obtained from Cognis, Ireland. Metal concentrations were determined by using Atomic Absorption Spectroscopy (AAS). The chemicals used were of AR grade.

\subsection{Experimental Procedure}

The feed solution for copper and nickel separation was prepared so as to contain $3.5 \mathrm{~g} / \mathrm{l}$ of copper and $0.35 \mathrm{~g} / \mathrm{l}$ of nickel in sulfate medium. LIX $664 \mathrm{~N}$ in kerosene was used as the organic phase. The maximum copper loading mentioned in the specifications for LIX 664N was 5.5 to $5.9 \mathrm{~g} / \mathrm{l}$ of copper. Hence feed solution with copper concentration lower than the maximum loading concentration was taken. The required amount of copper sulfate and nickel sulfate were dissolved in de-mineralized water to prepare the feed solution with concentration mentioned above. Literature states that copper (II) is strongly extracted at a $\mathrm{pH}$ of approximately 2 and nickel (II) at a higher $\mathrm{pH}$ of 9 to 9.5. Experiments were carried out by contacting the aqueous and organic phases using separating funnel at room temperature.

Kinetics of copper and nickel extraction was studied separately for both copper and nickel with a phase ratio of $1: 1$. The effect of the extractant, LIX \% (v/v) in kerosene on selectivity has been found by taking a mixture of copper and nickel sulfate solution with the ratio of copper to nickel as 10:1 and O:A as 2:1 at $\mathrm{pH}$ 2. The LIX percent that gave the highest selectivity is the desired \%LIX for separation of copper and nickel at $\mathrm{pH} 2$. Also at $\mathrm{pH} 2$, the influence of \%LIX on extraction of copper was found by contacting the aqueous feed solution with varying concentration of LIX (v/v) in kerosene.

With maximum amount of copper getting extracted at this $\mathrm{pH}$ of 2, the raffinate phase has nickel remaining in it. The $\mathrm{pH}$ of the raffinate was raised to 9 by adding ammonia solution and the influence of extractant LIX \% (v/v) on nickel extraction was studied. Extraction isotherm was developed for copper extraction and the number of stages required for copper extraction determined for both $\mathrm{O}: \mathrm{A}$ as $1: 1$ and 2:1.

Stripping of copper loaded organic phase was accomplished with relatively strong acid solutions. In this case it was done with $180 \mathrm{~g} / \mathrm{H}_{2} \mathrm{SO}_{4}$ with O:A as 1:1 in two stages.

Each time the raffinate concentration is found using AAS and the corresponding organic phase concentration of copper or nickel is found by the difference of the feed and the raffinate phase concentrations.

\section{Results}

The extractant, LIX chemically bonds to the metal ion at two sites and releases a hydrogen ion into the aqueous solution from which the copper is extracted in exchange for hydrogen ion. If the aqueous solution is relatively stronger in acid concentration; at $180 \mathrm{~g} / \mathrm{l}$ for example, the exchange of copper is in the other direction. Now the extractant releases the copper ions for hydrogen ions. This exchange is stripping.

Extraction can be described as:

$$
2 \mathrm{HR}_{\text {org }}+\mathrm{Cu}^{2+}{ }_{\text {aq }} \rightleftharpoons \mathrm{CuR}_{2 \text { org }}+2 \mathrm{H}^{+}{ }_{\text {aq }}
$$

Here HR is the extractant and aq. and org. are the 
aqueous and organic phases, respectively.

The extraction equilibrium constant, $K_{e x}$ is given as

$$
K_{e x}=\frac{\left[\mathrm{CuR}_{2}\right]_{\mathrm{org}}\left[\mathrm{H}^{+}\right]_{\mathrm{aq}}^{2}}{[\mathrm{Cu}]_{\mathrm{aq}}[\mathrm{HR}]_{\text {org }}^{2}}
$$

Stripping can be described as:

$$
\mathrm{CuR}_{2 \text { org }}+2 \mathrm{H}_{\text {aq }}^{+} \rightleftharpoons 2 \mathrm{HR}_{\text {org }}+\mathrm{Cu}_{\text {aq }}^{2+}
$$

Several factors such as the concentration of extractant, $\mathrm{pH}$ of aqueous phase, and temperature could have influence on the extraction of metal ions.

Figure 1 shows that the kinetics of copper extraction is very fast and the equilibrium is reached faster for copper extraction as compared to that for nickel. The \% extraction and distribution coefficient of copper increased with LIX \% as shown in Figures 2 and 3.

Selectivity gives the tendency of separation between

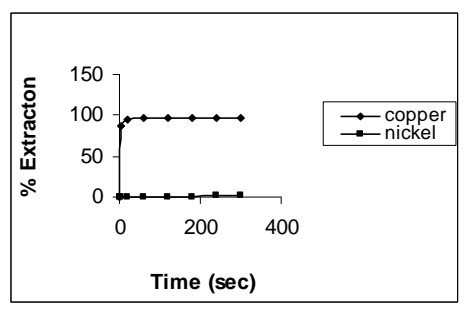

Figure 1. Kinetics of copper and nickel extraction with $\mathbf{3 0} \%$ LIX (v/v).

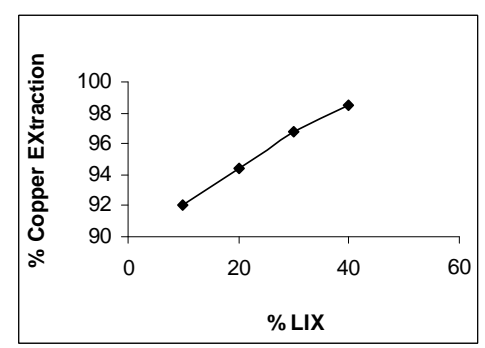

Figure 2. \% extraction of copper at different \% LIX (v/v) with $\mathrm{O}: \mathrm{A}=2: 1$.

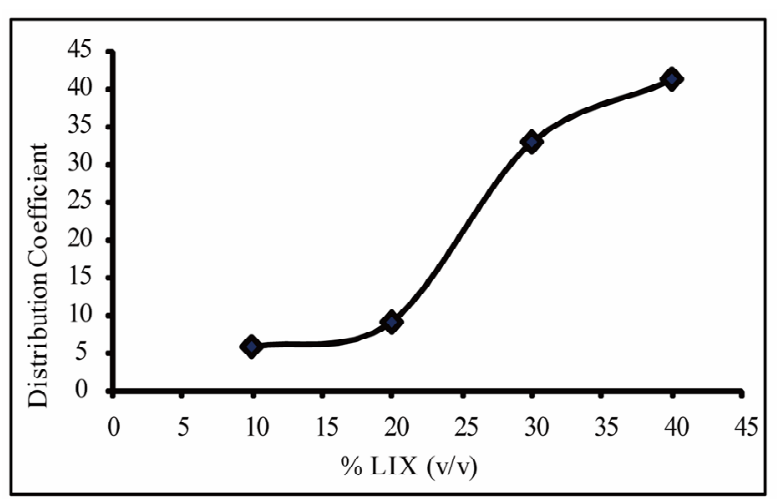

Figure 3. Distribution coefficient for copper. two species. Here it is the ratio of distribution coefficient of copper to that of nickel. For experiments performed on mixture of copper and nickel, the selectivity was as high as 6000 at $40 \%$ LIX (v/v). This is seen in Figure 4. The $\%$ extraction of copper and selectivity increased with increasing concentration of LIX. However, very high LIX concentration can have serious problems in extraction due to high viscosity and low mass transfer rates.

Extraction isotherm for copper is shown in Figure 5. The number stages determined for $\mathrm{O}: \mathrm{A}$ as $1: 1$ and 2:1 are shown in the figure. It shows that for the phase ratio O:A as $1: 1$, almost complete recovery of copper is possible in 2 stages. With O:A as 2:1, copper can be recovered almost completely in one stage. However 2 stages are recommended for $99.9 \%$ recovery. Thus LIX 664N shows very good extraction properties for copper.

The copper loaded organic phase was subjected to stripping with $180 \mathrm{~g} / \mathrm{l} \mathrm{H}_{2} \mathrm{SO}_{4}$ at O:A phase ratio of $1: 1$ in two stages. Overall copper recovered from the organic phase in the stripping stage is $98.5 \%$ with the recovery as $86 \%$ and $12.5 \%$ respectively in the two stages.

For extraction of nickel remaining in the raffinate phase after extraction of copper, the effect of \% LIX $664 \mathrm{~N}(\mathrm{v} / \mathrm{v})$ as solvent was found on \% extraction of nickel at $\mathrm{pH}$ 9. Maximum \% extraction was approximately $80 \%$ at $30 \%$ LIX (v/v) as seen in Figure 6. These values are not as high as those observed for copper.

\section{Conclusions}

The present study shows that 30\% LIX 664N in kerosene

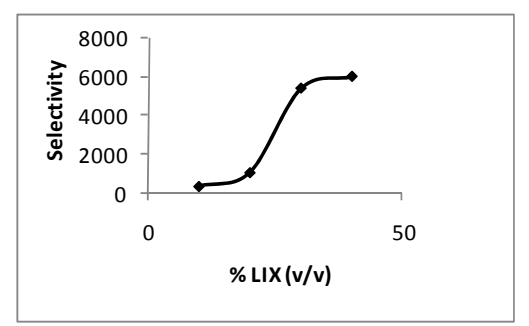

Figure 4. Selectivity of copper over nickel.

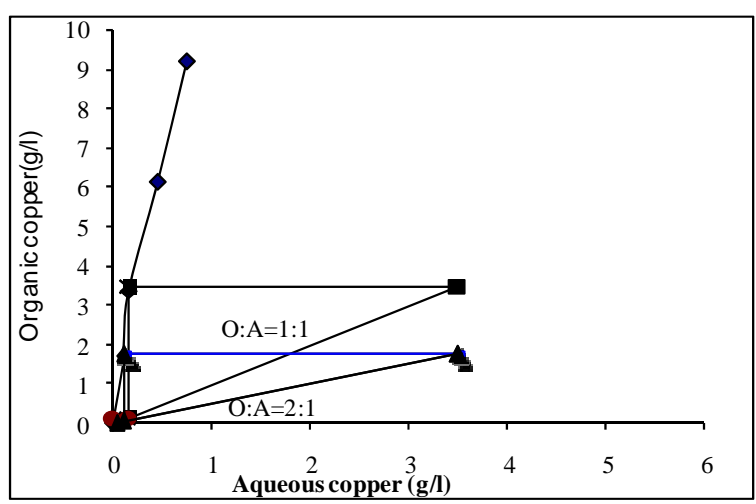

Figure 5. Extraction isotherm of copper. 


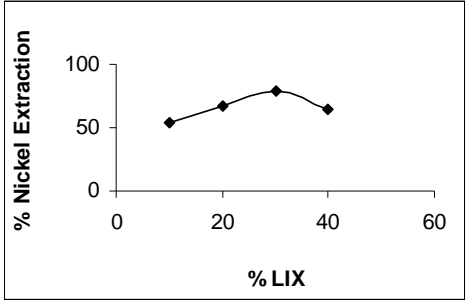

Figure 6. \% extraction of nickel at different \% LIX (v/v).

at $\mathrm{pH} 2$ has excellent extraction properties for recovery of copper. Not only is the selectivity very high for extraction of copper versus nickel, the kinetics is also favorable for copper extraction. The distribution coefficient for copper at these conditions is also very high. Thus the two metals, copper and nickel, can be easily separated.

Studies for stripping of copper from the organic phase using $180 \mathrm{~g} / \mathrm{l}$ sulfuric acid showed that $98.5 \%$ copper can be recovered from solvent phase. This would permit the recycle of copper-stripped solvent for further use in extraction stage of the process.

At pH 9 and 30\% LIX 664N in kerosene, up to 80\% nickel can be recovered from the raffinate phase after recovery of copper.

\section{Acknowledgements}

We would like to thank the management of Mukesh Patel School of Technology Management \& Engineering (NMIMS), Mumbai for supporting the work. We thankfully acknowledge Mr. Manish Kapadia (BS International, Mumbai) for supplying LIX 664N. Our gratitude to Mr. D. Gokani (Consultant) for his valuable suggestions.

\section{REFERENCES}

[1] S. Zhang and E. Forssberg, "Mechanical SeparationOriented Characterization of Electronic Scrap,” Resource, Conservation and Recycling, Vol. 21, No. 4, 1997, pp. 247-269. doi:10.1016/S0921-3449(97)00039-6

[2] B. Kim, J. Lee, S. Seo Y. Park and H. Sohn, “A Process for Extracting Precious Metals from Spent Printed Circuit Boards and Automobile Catalysts," Journal of Management, Vol. 56, No. 12, 2004, pp. 55-58. doi:10.1007/s11837-004-0237-9
[3] M. Iji and S. Yokoyama, "Recycling of Printed Wiring Boards with Mounted Electronic Components," Circuit World, Vol. 23, No. 3, 1997, pp. 10-15. doi:10.1108/03056129710370196

[4] J. A. S. Williams, "A Review of Electronics Demanufacturing Processes,” Resources Conservation and Recycling, Vol. 47, No. 3, 2006, pp. 195-208. doi:10.1016/j.resconrec.2005.11.003

[5] R. W. Gibson, P. D. Goodman, L. Holt, I. M. Dalrymple and D. J. Fray, "Process for the Recovery of Tin, Tin Alloys or Lead Alloys from Printed Circuit Boards," US Patent 6641712, 2003.

[6] C. Hagelüken, "Recycling of Electronic Scrap at Umicore's Integrated Metals Smelter and Refinery," Proceedings of European Metallurgical Conference, Dresden, 13-21 September 2005, pp. 152-161.

[7] Y. J. Park and D. J. Fray, "Recovery of High Purity Precious Metals from Printed Circuit Boards," Journal of Hazardous Materials, Vol. 164, No. 2-3, 2009, pp. 11521158. doi:10.1016/j.jhazmat.2008.09.043

[8] Y. J. Park and D. J. Fray, "Separation of Zinc and Nickel Ions in a Strong Acid through Liquid-Liquid Extraction," Journal of Hazardous Materials, Vol. 163, No. 1, 2009, pp. 259-265. doi:10.1016/j.jhazmat.2008.06.085

[9] C. R. Merigold, "LIX Reagent Solvent Extraction Plant Operating Manual for Small and Medium Size LeachSolvent Extraction-Electro Winning Copper Recovery," Operations by Henkel Corporation, Minerals Industry Division, Tucson, 1996.

[10] F. J. Alguacil and A. Cobo, "Solvent Extraction with LIX 973N for the Selective Separation of Copper and Nickel," Journal of Chemical Technology and Biotechnology, Vol. 74, No. 5, 1999, pp. 467-471. doi:10.1002/(SICI)1097-4660(199905)74:5<467::AID-JC TB60>3.0.CO;2-7

[11] V. Sridhar, J. K. Verma and S. A. Kumar, "Selective Separation of Copper and Nickel by Solvent Extraction Using LIX 984N,” Hydrometallurgy, Vol. 99, No. 1-2, 2009, pp. 124-126. doi:10.1016/j.hydromet.2009.07.007

[12] D. S. Flett and J. Melling, "Extraction of Ammonia by Commercial Copper Chelating Extractants,” Hydrometallurgy, Vol. 4, No. 2, 1979, pp. 135-146. doi:10.1016/0304-386X(79)90042-2

[13] I. Giannopoulou and D. Panias, "Copper and Nickel Recovery from Acidic Polymetallic Aqueous Solutions," Minerals Engineering, Vol. 20, No. 8, 2007, pp. 753-760. doi:10.1016/j.mineng.2007.02.009 